\title{
Searching for Quark Matter at the CERN SPS
}

\author{
C. Lourenço \\ CERN-EP, CH-1211 Geneva 23, Switzerland
}

\begin{abstract}
This article gives a brief overview of some recent advances in our understanding of the physics of dense strongly interacting matter, from measurements done at the CERN SPS. The presently available results are very interesting, and are likely to reflect the production of a new state of matter in central $\mathrm{Pb}-\mathrm{Pb}$ collisions, at the highest SPS energies. However, important questions require further work. Particular emphasis is given to developments made since the Quark Matter 1999 conference, and to issues that justify the continuation of the SPS heavy ion physics program beyond year 2000 .
\end{abstract}

Invited talk presented at the Quark Matter 2001 conference

Stony Brook, USA, January 14-20, 2001

To be published in the Proceedings 



\title{
Searching for Quark Matter at the CERN SPS
}

\author{
Carlos Lourenço
}

CERN-EP, Geneva, Switzerland

This article gives a brief overview of some recent advances in our understanding of the physics of dense strongly interacting matter, from measurements done at the CERN SPS. The presently available results are very interesting, and are likely to reflect the production of a new state of matter in central $\mathrm{Pb}-\mathrm{Pb}$ collisions, at the highest SPS energies. However, important questions require further work. Particular emphasis is given to developments made since the Quark Matter 1999 conference, and to issues that justify the continuation of the SPS heavy ion physics program beyond year 2000.

\section{Introduction}

The study of high energy heavy ion collisions is presently a very active field in experimental particle physics, with the RHIC collider in operation at BNL since summer 2000 and with the ALICE experiment being prepared to study this kind of physics at the LHC energies. The first goal of this experimental program, which started in 1986, with the AGS and SPS fixed-target programs, is the discovery of the phase transition from confined hadronic matter to deconfined partonic matter. The idea that such a phase transition should exist, between hadronic and quark matter, is around since the first models of the quark structure of hadrons. It is presently studied in detail in the framework of Lattice QCD calculations, which predict its occurrence when the temperature of the system exceeds a critical threshold at around $170 \mathrm{MeV}$, corresponding to a critical energy density of around $700 \mathrm{MeV} / \mathrm{fm}^{3}$ [1]. The proof of existence of the quark matter phase and the study of its properties are key issues in QCD, for the understanding of confinement and chiral-symmetry.

When this new state of matter was postulated, some signatures of its formation in high energy nuclear collisions were proposed, on the basis of theoretical arguments, among which we can highlight the enhancement of strange particle production, the suppression of charmonia states $\left(\mathrm{J} / \psi, \chi_{c}\right.$ and $\left.\psi^{\prime}\right)$, due to the screening of the $c \bar{c}$ binding potential in the QGP colour soup, and the production of thermal dileptons, electromagnetic radiation emitted by the 'free' quarks. The results obtained by the SPS experiments, after 15 years of collecting data with proton and ion beams, provide "compelling evidence for the existence of a new state of matter, in which quarks roam freely", produced in central $\mathrm{Pb}-\mathrm{Pb}$ collisions at the highest SPS energies. Among the most exciting observations are the enhanced production of multistrange hyperons, the centrality dependence of the $\mathrm{J} / \psi$ suppression pattern and the enhancement of intermediate mass dimuon production, a possible indication of thermal dimuons. Besides, the enhanced production of low mass dileptons may be an indication of approach to chiral symmetry restoration. 
In view of these exciting results and in order to clarify important questions remaining open, a new experiment, NA60, has been approved at CERN, extending the SPS runs with heavy ion beams and bridging the gap between the original SPS program and the future high-energy wonderland of ALICE. At the other end of the energy scale, the NA49 experiment will have a ten day extension with $\mathrm{Pb}$ ions at 20 and $30 \mathrm{GeV}$ per nucleon, to complete the energy scan of global strangeness production. So far it has collected data at 158, 40 and $80 \mathrm{GeV}$ per nucleon. This extension of the SPS heavy ion running time will bring to a proper conclusion the program started in 1986, whilst providing valuable information, complementary to the studies underway at RHIC.

This paper gives a somewhat personal overview of the present status of the SPS results, briefly mentions the progress made since the last Quark Matter conference, presents some pertinent questions that remain open, and dreams about how the future SPS program could address those issues.

\section{Present status of the SPS results}

The very large amount of experimental results obtained by the CERN SPS experiments since 1986 is so vast and diversified that a proper review would require a much more extensive article, jointly prepared by several of the active players in the field. The previous conference in the Quark Matter series, the last one before the RHIC experiments started collecting data, ended with two summary talks that reviewed in detail the status of the field in terms of hadronic [2] and dilepton [3] signals. A few attempts have also been made to see in a coherent way some of the most significant results, in particular those obtained with the lead beam at the SPS [4]. A tentative summary has been proposed [5], basically saying that "the combined results provide compelling evidence for the existence of a new state of matter, featuring many of the characteristics of the primordial soup in which quarks and gluons existed before they clumped together as the universe cooled down". The present paper is too short, and is not the right place, for critically commenting the scientific meaning (and opportunity) of these words.

It is certainly appropriate to say that all the CERN SPS experiments have been successful in delivering significant information, many of them having seen "what they were looking for". However, these 15 years have also confirmed that heavy ion collisions lead to very complicated (and fastly evolving) systems, and that it is difficult to extract clear messages from the observations. Central collisions between two $\mathrm{Pb}$ nuclei, at the highest SPS energies, lead to the production of hundreds of final-state particles widely emitted without discernible structures. These complex, and apparently chaotic, final states can be studied applying statistical concepts, attempting descriptions based on (non-perturbative) QCD thermodynamics, and summarized by macroscopic variables like temperature, pres-

sure, etc. But such studies present formidable challenges, requiring complex (and quite expensive) experimental techniques, demanding huge amounts of computing time, and leading, after a major effort of many people, to a few points on a figure. The usefulness of such measurements must be evaluated by their ability to rule out existing alternative models or, at least, by their power in constraining assumptions and free parameters.

One of the most important lessons from the SPS program has been, in fact, that it is extremely difficult to rule out "continuously improving models" that are always able to reproduce, a posteriori, any new measurements. In these conditions, the fact that "we have seen what we were looking for", does not mean that we have made any progress in 
our understanding. Twenty years after the first Quark Matter conferences, and after a considerable evolution in the theoretical and experimental sectors of this field, we still wonder, frequently, if we really know "what we are looking for". In spite of the strong indications that very interesting phenomena occur in the early stages of a $\mathrm{Pb}-\mathrm{Pb}$ collision, at the highest SPS energies, we still do not know the answer to the critical question that motivates this field: can we convince ourselves and the community at large that we have formed quark matter in the laboratory?

In our field, as in most scientific enterprises, each step forward brings new questions, at the same time as it solves older ones. The value of a scientific discovery is, also, in the relevance of the new questions it raises. But have we really solved any question so far? How many models, that clearly fail to properly reproduce already established results, using parameters within reasonable ranges, have been definitely ruled out? Once more, in this conference, we have seen old models, with new free parameters, in ridiculous exercises attempting to describe experimental measurements, bluntly ignoring their (small) error bars, and just succeeding in increasing the level of entropy in the field. We should be happy when our models are successfully falsified. As Bo Andersson said in his Quark Matter 1985 paper [6], quoting Bacon, "nature never tells you when you are right but only when you are wrong. Therefore, you have only learned something when you disagree with the data. For some reason, the scientific society does not seem to honour the successfully wrong theorists". While looking for 'critical phenomena' in nature, we must follow a critical behaviour in our own work, both in the measurement and in the interpretation steps. "Whenever we propose a solution to a problem, we ought to try as hard as we can to overthrow our solution, rather than defend it. Few of us, unfortunately, practice this precept; but other people, fortunately, will supply the criticism for us" [0].

The final clarification of the present SPS results requires a careful and systematic approach, to establish beyond reasonable doubt that the QCD phase transition from hadronic to quark matter happens in central $\mathrm{Pb}-\mathrm{Pb}$ collisions at the highest SPS energies. The available measurements should be critically scrutinized, looking for possible biases of any kind. In cases where information is obviously missing, new measurements should be urgently done. Models that pretend to explain the available results must provide specific predictions for future measurements, with appropriate and carefully explained uncertainty bands. If the new observations validate those predictions, we will have, then, made substantial progress. Otherwise, without a devoted and objective effort, seriously joining theory and experiment, the epitaph of the SPS heavy ion program will be "just when we were about to find the answer, we forgot the question".

\section{Strangeness production}

One of the earliest predictions in the field is that particles containing strange quarks should be produced more often if the system produced in the heavy ion collisions passes through a quark-gluon plasma phase. A global increase of around a factor 2 in strangeness production (dominated by kaon production), has indeed been observed, in particular by the NA49 large-acceptance experiment. The most spectacular observations have been done, however, in the multi-strange hyperon sector. The very large enhancement factors in particle yields per participating nucleon, reaching a factor around 17 for the $\Omega$, a triple strange hyperon, and the fact that these factors are significantly higher for the states with more strange quarks, i.e. $E_{\Omega}>E_{\Xi}>E_{\Lambda}$, where $E_{i}$ is the enhancement of the 
particle $i$ with respect to $\mathrm{p}-\mathrm{A}$ interactions, are naturally explained if the particle yields are determined from statistical hadronization of a strangeness-enhanced plasma phase. On the contrary, such enhancement levels cannot be reproduced in conventional (final state hadronic rescattering) scenarios, given the short lifetime of the expanding hadronic system (see, however, Ref. [8]). When these results were presented in the Quark Matter 1999 conference [9], a question was left in the air: is there a threshold behaviour in the enhancement pattern, between the $\mathrm{p}$ - $\mathrm{Be}$ and $\mathrm{p}-\mathrm{Pb}$ points and the $\mathrm{Pb}-\mathrm{Pb}$ values? The flat pattern observed in the $\mathrm{Pb}-\mathrm{Pb}$ data indicated very little dependence on the centrality of these collisions, for $N_{\text {part }}>100$. Where was the transition?

The NA57 experiment has continued these studies, making a special effort to collect peripheral $\mathrm{Pb}-\mathrm{Pb}$ collisions. The first results, presented at this conference [10], show that the enhancement of $\bar{\Xi}^{+}$production increases by a factor 2.6 , from $N_{\text {part }}=62$ to 121 . The confirmation of a threshold behaviour in the strangeness enhancement pattern may come from the data analysis of the other strange hyperons. Unfortunately, having only five bins in centrality, and four of them showing a flat behaviour, the $\mathrm{Pb}-\mathrm{Pb}$ pattern measured by the NA57 experiment will fall short of showing a clear transition, with a characteristic threshold, if it exists. A better understanding of the apparent onset of the enhancement would require studying "intermediate mass" nuclear collisions, like In-In, for instance, in small centrality steps. Unfortunately, the effort required by the preparation of the ALICE experiment seems to prevent the realisation of such future studies.

Another major addition to the strangeness chapter is being provided by the NA49 experiment, when running with proton beams. The very large acceptance of this experiment makes it particularly appropriate in the study of asymmetric collision systems, as p-A interactions, where the reflection of the probed phase space window around midrapidity cannot be performed. First results of "NA49-hadrons" have been shown at this conference [11], raising some questions on the "p-A reference baseline" used by WA97/NA57 in the extraction of the enhancement factors. Fortunately, we will see further data on this issue in the near future, since "NA49-hadrons" has been approved for further running in the next few years, and NA57 will collect more data on proton induced collisions in 2001 .

Still in the strangeness sector, long standing questions concerning $\phi$ production remain unclear. NA50 sees, in the dimuon decay channel, a strong increase in the yield of $\phi$ mesons produced in heavy ion collisions and a transverse mass spectrum with a rather low "inverse slope" [12], contrary to the observations of the NA49 experiment [13], in the $\mathrm{K}^{+} \mathrm{K}^{-}$decay channel. Future measurements of low- $p_{\mathrm{T}} \phi$ production in the dilepton channel, by NA60, should help clarifying the source of discrepancy.

\section{Low mass dilepton production}

The CERES experiment has observed that the yield of low mass $e^{+} e^{-}$pairs measured in $\mathrm{p}-\mathrm{Be}$ and $\mathrm{p}-\mathrm{Au}$ collisions is properly described by the expected "cocktail" of hadronic decays, while in $\mathrm{Pb}-\mathrm{Au}$ collisions, on the contrary, the measured yield, in the mass region 0.2-0.7 GeV, exceeds by a factor 2.5 the expected signal [14]. These observations are consistent with the expectation that the properties of vector mesons should change when produced in dense matter. In particular, near the phase transition to the quark-gluon phase, chiral symmetry should be partially restored, making the vector mesons indistinguishable from their chiral partners, thereby inducing changes in their masses and decay widths. The short lifetime of the $\rho$ meson, shorter than the expected lifetime of the dense 
system produced in the SPS heavy ion collisions, makes it a sensitive probe of medium effects and, in particular, of chiral symmetry restoration.

Already E. Shuryak [15] and B. Müller [16], in their Quark Matter 1999 papers, emphasized the importance of a considerable improvement in the CERES measurements of low mass dilepton production, in terms of signal to background ratio, mass resolution, and statistics. A TPC was added to the CERES setup, to improve the momentum resolution of the dielectron measurement. Unfortunately, problems in the data taking during year 1999 have prevented the CERES experiment from collecting a reasonable sample of dilepton events [17]. Those problems were solved in time for the run of year 2000, but a first look into this new data sample indicates that the anticipated performance has not been fully reached, at least in what concerns mass resolution and statistics. An accurate measurement of the $\omega$ resonance, necessary as a reference in the studies of the in-medium modifications apparently affecting the $\rho$, may remain in the wish list until the first runs of the NA60 experiment.

\section{Intermediate mass dilepton production}

The NA38 and NA50 experiments have studied the production of dileptons in the mass window between the $\phi$ and the $\mathrm{J} / \psi$ peaks, as a superposition of Drell-Yan dimuons and simultaneous semileptonic decays of $D$ and $\bar{D}$ mesons, after subtraction of the combinatorial background from pion and kaon decays [18]. The dimuon mass spectra measured in p-A collisions are very well reproduced taking the high mass region to normalize the Drell-Yan component and an open charm cross-section in good agreement with direct measurements made by other experiments. On the contrary, the superposition of Drell-Yan and open charm contributions, with the nucleon-nucleon absolute cross sections scaled with the product of the mass numbers of the projectile and target nuclei (as expected for hard processes), fails to properly describe the dimuon yield measured in ion collisions.

The data can be properly reproduced by simply increasing the open charm yield, with a scaling factor that grows linearly with the number of nucleons participating in the collision, reaching a factor 3 in the most central $\mathrm{Pb}-\mathrm{Pb}$ collisions. The observed excess can also be due to the production of thermal dimuons, a signal that was the original motivation for the NA38 experiment. In particular, the intermediate mass dimuons produced in the most central $\mathrm{Pb}-\mathrm{Pb}$ collisions are well reproduced by adding thermal radiation, calculated according to the model of Ref. [19], to the Drell-Yan and charm contributions normally extrapolated from nucleon-nucleon collisions. This model explicitly includes a QGP phase transition with a critical temperature of $175 \mathrm{MeV}$. The best description of the data is obtained using $\sim 250 \mathrm{MeV}$ as the initial temperature of the QGP medium radiating the virtual photons. The presently available data cannot distinguish between an absolute enhancement of charm production and the emission of thermal dilepton radiation. The clarification of the nature of the physics process behind the observed excess is also in the wish lists presented by E. Shuryak and B. Müller in their Quark Matter 1999 papers, and is the strongest physics motivation of the NA60 experiment.

\section{Charmonia production and suppression}

The formation of a deconfined medium should induce a considerable suppression of the charmonia production rate, due to the colour 'Debye' screening of the $c \bar{c}$ potential or to 
the breaking of the $c \bar{c}$ binding by scattering with energetic (deconfined) gluons. However, even the relatively simple measurement of $\mathrm{J} / \psi$ production faces a big challenge when it comes to furnish a convincing logical case that proves, to the satisfaction of the experts in the field, that a deconfined state of matter has been formed. It is not enough to show that a certain observable changes from $\mathrm{p}-\mathrm{Pb}$ to $\mathrm{Pb}-\mathrm{Pb}$ collisions, for instance, or to argue that its value in the most central nucleus-nucleus collisions is different from what is calculated in a 'conventional physics' model. The best path to clearly establish a solid result and shed light in this complicated field is to build a robust set of measurements, that establishes a precise reference baseline, relative to which the specific behaviour of heavy ion collisions can be extracted. Such a baseline shows what is the 'normal' behaviour of the signal we are studying, with respect to which we look for changes due to QGP formation. Furthermore, we are in a much better position if nature provides us with a reference process, insensitive to the formation of a deconfined phase, specially if we can measure it with the same detector.

In the case of the $\mathrm{J} / \psi$ suppression topic, the baseline is built from the measurements done by NA38 and NA50 with pp, p-A and light ion collisions [20]. Very peripheral Pb$\mathrm{Pb}$ collisions have been successfully collected in year 2000 and we will soon know how well they follow the "normal nuclear absorption" baseline. The best reference physics process, at SPS energies, is the rate of high mass Drell-Yan dimuons, seen to scale with the number of binary nucleon collisions, up to the $\mathrm{Pb}-\mathrm{Pb}$ case. It is well known that the $\mathrm{J} / \psi$ production yield, in $\mathrm{Pb}-\mathrm{Pb}$ collisions, shows a very peculiar behaviour as a function of the centrality of the collisions, estimated either using the transverse or the forward energies [21]. The observed two-step suppression pattern is in clear disagreement with the predictions of 'conventional' models, which attribute the disappearance of the $\mathrm{J} / \psi$ mesons to interactions with 'comoving' hadrons, while it is naturally explained if the charmonia states are dissolved in a deconfined medium, due to the different melting temperatures of the $\chi_{c}$ and $\mathrm{J} / \psi$ states (about $30-40 \%$ of the observed $\mathrm{J} / \psi$ mesons result from the decay of $\chi_{c}$ states).

If we accept that this pattern indicates the production of a state of matter where colour is no longer confined, we must move on to the detailed understanding of how deconfinement sets in, and what physics variable governs the threshold behaviour of the $\left(\chi_{c}\right)$ suppression: (local) energy density, density of wounded nucleons, of percolation clusters, of produced gluons, etc. This requires collecting data with a smaller nuclear collision system like In-In. Indeed, it is possible to predict at which impact parameter, $b$, of In-In collisions is reached the same threshold in (local) energy density, or any other variable, as reached in $\mathrm{Pb}-\mathrm{Pb}$ collisions of $b \approx 8 \mathrm{fm}$, where the $\chi_{c}$ state starts melting. A verification of such specific predictions would be the final element of proof that the deconfined quark-gluon phase sets in, and would provide fundamental information on the mechanisms behind the observed phenomena. In this context, it is also important to improve our knowledge of the nuclear dependence of $\chi_{c}$ production, in $\mathrm{p}-\mathrm{A}$ collisions, at SPS energies.

The $\mathrm{J} / \psi$ data do not provide a direct measurement of the critical temperature. Finite temperature lattice QCD tells us that the strongly bound $\mathrm{J} / \psi c \bar{c}$ state should be screened when the medium reaches temperatures 30-40\% higher than $T_{c}$, while the larger and more loosely bound $\psi^{\prime}$ state should melt near $T_{c}$. The $\psi^{\prime}$ is already significantly suppressed when going from $\mathrm{p}-\mathrm{U}$ to peripheral $\mathrm{S}-\mathrm{U}$ collisions but the presently existing results are not clear 
in what concerns the pattern of the $\psi^{\prime}$ suppression. Is the "anomalous" $\psi^{\prime}$ suppression due to QGP melting or to hadronic absorption? If we see that this suppression happens more or less in an abrupt way, within a single collision system rather than comparing p- $\mathrm{U}$ to $\mathrm{S}-\mathrm{U}$ data, we would know that Debye screening is the mechanism responsible for the $\psi^{\prime}$ disappearance and we would have a clear measurement of $T_{c}$. This requires a new measurement, with improved mass resolution to have a cleaner separation between the $\psi^{\prime}$ and $\mathrm{J} / \psi$ peaks, and which scans an energy density region including the $\mathrm{p}-\mathrm{U}$ and the $\mathrm{S}-\mathrm{U}$ points.

Improved measurements of $\mathrm{J} / \psi$ and $\psi^{\prime}$ production, with intermediate mass nuclei, were also included in the wish lists of E. Shuryak and B. Müller, and are an important part of the physics program of the NA60 experiment, which will also measure $\chi_{c}$ production in p-A collisions.

\section{Open charm production}

Knowing that the bound $c \bar{c}$ states are suppressed, it is natural to ask what happens to the unbound charm. Charm quarks are so heavy that they can only be produced at the earlier stages of the nuclear collision, before the eventual formation of the QGP state. Charm is the heaviest flavour that can be studied in heavy ion collisions at the SPS energies. The production of charm quarks leads mainly to correlated pairs of $D$ and $\bar{D}$ mesons. Only a few percent of the charmed quark pairs end up in the bound charmonia states presently studied by the NA50 experiment, and which exhibit a rather interesting "anomalous" behaviour. What happens to the vast majority of $c$ quarks? Are they affected by energy loss while crossing the dense (partonic or hadronic) medium? Is charm production enhanced similarly to what has been seen in the strangeness sector? Finally, $D$ meson production provides the natural reference with respect to which we should study the observed $\mathrm{J} / \psi$ suppression, since both production mechanisms depend on the same gluon distribution functions. If charm production is enhanced in nuclear collisions, it makes the $\mathrm{J} / \psi$ suppression even more anomalous. A direct observation of $D$ meson production is clearly the most important new measurement that remains to be done at the SPS, and constitutes a basic reason for the construction and running of NA60.

\section{Future prospects}

The results and open questions presented in the previous sections emphasize the importance of having a new experiment at the SPS, that can significantly improve several existing observations and make a few new measurements, including a measurement of open charm production in heavy ion collisions. A dedicated experiment is needed, that can cope with the very high particle multiplicities reached in the most central nuclear collisions (400 charged particles per unit rapidity at midrapidity) and with the rather small $D$ production cross section.

The NA50 experiment has been using CERN's highest intensity heavy ion beam (more than $10^{7}$ ions per second) and has a very selective dimuon trigger, quite appropriate to look for rare processes. The recently approved NA60 experiment [22], complements the muon spectrometer and zero degree calorimeter already used in NA50 with two state-of-the-art silicon detectors, placed in the target region: a radiation hard beam tracker, consisting of four silicon microstrip detectors placed on the beam and operated at a temperature 
of $130 \mathrm{~K}$, and a 10-plane silicon pixel tracking telescope, made with radiation tolerant readout pixel chips, placed in a $2.5 \mathrm{~T}$ dipole magnetic field.

The NA60 experiment has been approved to run from 2001 to 2003, using proton, $\mathrm{Pb}$ and In beams. The following questions summarize the physics motivation of NA60.

- What is the origin of the dimuon excess seen in the intermediate mass region? Thermal dimuon production?

- Is the open charm yield enhanced in nucleus-nucleus collisions? How does it compare to the suppression pattern of bound charm states?

- What is the variable (local energy density, cluster density, etc.) that rules the onset of charmonia suppression?

- What is the physical origin of the $\psi^{\prime}$ suppression? If it is due to Debye screening, what is its melting temperature?

- Which fraction of the $\mathrm{J} / \psi$ yield comes from $\chi_{c}$ decays? Does it change from $\mathrm{p}$-Be to $\mathrm{p}-\mathrm{Pb}$ collisions?

- Are there medium induced modifications in the $\rho$ meson? Is there a threshold behaviour in the low mass dilepton enhancement? What happens with the $\omega$ meson?

- Is the observed $\phi$ enhancement a specific feature of heavy ion collisions? Is the $\phi$ sensitive to flow?

The beam tracker gives the transverse coordinates of the interaction point, on the targets, with enough accuracy to measure the impact parameter of the muon tracks, i.e. the minimum distance between the track and the collision vertex, in the transverse plane. Thanks to this information, NA60 will be able to separately study the production of prompt dimuons and the production of muons originating from the decay of charmed mesons, in p-A and heavy ion collisions. The prompt dimuon analysis will use events where both muons come from (very close to) the interaction vertex. The open charm event sample is composed of those events where both muon tracks have a certain minimum offset with respect to the interaction point and a minimal distance between themselves at $z_{\text {vertex }}$. It should not be difficult to see which of these two event samples is enhanced by a factor of 2 or 3 in nuclear collisions of $N_{\text {part }} \sim 300$.

The high granularity tracking telescope, placed in a powerful dipole field, gives access to the muon tracks at the vertex level and vastly improves the mass resolution of the dimuon measurement. This has been demonstrated in a very fast feasibility test done in 1998, using a small telescope (four half-planes) made of the previous generation of readout pixel chips. The results, shown in Fig. 1, confirm that the mass resolution improves from 70 to $20 \mathrm{MeV}$ at the $\omega$ mass, as expected from the physics performance simulations.

The studies of prompt dimuon and open charm production in p-A collisions are important reference measurements, to understand the results obtained with nuclear collisions. In particular, the ratio between the open charm and the Drell-Yan production cross sections will be determined with high accuracy in several p-A collision systems, revealing if these two hard processes have the same A-dependence or not. More challenging will be the measurement of the dependence of $\chi_{c}$ production on the mass number of the target, in $\mathrm{p}$-A collisions, by seeing how the ratio between $\chi_{c}$ and $\mathrm{J} / \psi$ yields changes from $\mathrm{p}$-Be to $\mathrm{p}-\mathrm{Pb}$ collisions. To minimize the systematical uncertainties due to the beam flux normalization, the measurement will be done using the $\mathrm{Be}$ and $\mathrm{Pb}$ targets simultaneously in the beam. The $\chi_{c} \rightarrow \psi \gamma \rightarrow \psi e^{+} e^{-}$decays will be used for this study, with the photons 

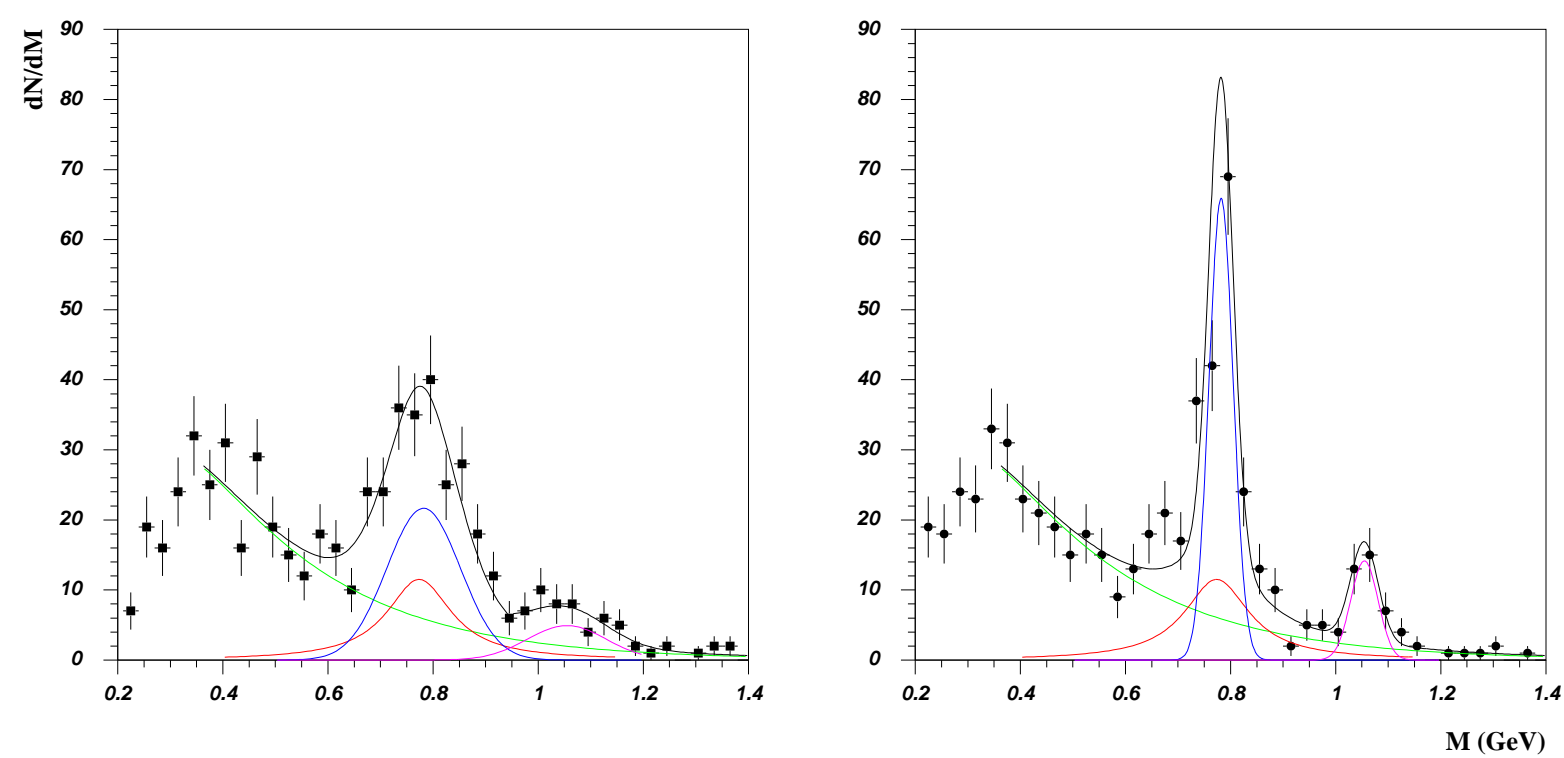

Figure 1. Dimuon mass distributions measured in 1998, in p-Be collisions, before (left) and after (right) using the information of the test pixel telescope. The curves represent the low mass vector meson resonances $(\rho, \omega$ and $\phi)$ on the top of a continuum. They are normalized to the same number of events in both figures. The collected statistics (600 events) correspond to a few minutes of NA60 running.

converting in a $\mathrm{Pb}$ disk placed downstream of the targets, and the electron-positron pairs reconstructed in the silicon pixel telescope.

\section{Summary and conclusions}

Starting from the questions and wishes expressed at the Quark Matter 1999 conference, concerning measurements that should be done at the CERN SPS before closing this facility, I have briefly mentioned some of the most recent developments and emphasized the issues that have imposed a continuation of the SPS heavy ion physics program.

After 15 years of "learning curve", we can say that we have been unable to falsify the hypothesis of quark gluon plasma formation at the CERN SPS. In fact, as predicted, strangeness is enhanced, the $\mathrm{J} / \psi$ is suppressed, the dimuon continuum looks as if thermal dileptons are produced, and there are modifications in the low mass dilepton spectra, among other observations. These results provide extremely relevant information about the (predicted) formation of a deconfined state of matter in high energy heavy ion collisions. However, considerable homework remains to be done in view of converting "compelling evidence" into "conclusive evidence" that the quark matter phase has indeed been formed at CERN. This is exactly the reason why the heavy ion community must make a significant effort to further clarify the present results and reach a deeper understanding of the critical behaviour of QCD at SPS energies.

The re-birth of the heavy ion physics program at the CERN SPS, with the extension of NA49 and the approval of the new NA60 experiment, represents an evolution from a broad physics program to a dedicated study of specific signals that already provided very 
interesting results. It is not clear that the NA60 experiment will run successfully, given the delays in the availability of pixel readout chips and the dramatic lack of resources (people and budget). If it does, the new measurements should give a significant contribution to the understanding of the presently existing results, and considerably help in building a convincing logical case that establishes beyond reasonable doubt the formation (or not) of a deconfined state of matter in heavy ion collisions at the SPS.

\section{REFERENCES}

1. F. Karsch, these proceedings.

2. R. Stock, Proc. of QM'99, Nucl. Phys. A 661 (1999) 282c.

3. L. Kluberg, Proc. of QM'99, Nucl. Phys. A 661 (1999) 300c.

4. R. Stock, Phys. Lett. B 456 (1999) 277

U. Heinz, Proc. of QM'99, Nucl. Phys. A 661 (1999) 140c.

5. M. Jacob and U. Heinz, CERN press release, Feb. 2000.

6. B. Andersson, Proc. of QM'85, Nucl. Phys. A 447 (1985) $165 \mathrm{c}$.

7. K. Popper, "The Logic of Scientific Discovery", 1959.

8. K. Redlich, these proceedings.

9. F. Antinori et al. (WA97 Coll.), Proc. of QM'99, Nucl. Phys. A 661 (1999) 130c.

10. N. Carrer et al. (NA57 Coll.), these proceedings.

11. T. Susa et al. (NA49 Coll.), these proceedings.

12. N. Willis et al. (NA50 Coll.), Proc. of QM'99, Nucl. Phys. A 661 (1999) 534c.

13. V. Friese et al. (NA49 Coll.), these proceedings.

14. B. Lenkeit et al. (CERES Coll.), Proc. of QM'99, Nucl. Phys. A 661 (1999) 23c.

15. E. Shuryak, Proc. of QM'99, Nucl. Phys. A 661 (1999) 119c.

16. B. Müller, Proc. of QM'99, Nucl. Phys. A 661 (1999) 272c.

17. H. Appelshauser et al. (CERES Coll.), these proceedings.

18. L. Capelli et al. (NA50 Coll.), these proceedings.

19. R. Rapp and E. Shuryak, Phys. Lett. B 473 (2000) 13.

20. E. Scomparin et al. (NA50 Coll.), these proceedings.

21. P. Bordalo et al. (NA50 Coll.), these proceedings.

22. A. Baldit et al. (NA60 Coll.), Proposal CERN/SPSC 2000-010, March 2000. 